\title{
On the Hyperbolicity of Two- and Three-Layer Shallow Water Equations
}

\author{
Manuel Castro ${ }^{\dagger}$, Jörn Thies Frings ${ }^{\ddagger}$, Sebastian Noelle ${ }^{\ddagger}$, \\ Carlos Parés ${ }^{\dagger}$, Gabriella Puppo*
}

Bericht Nr. $314 \quad$ Dezember 2010

Key words: Multi-layer shallow water equations, Kelvin-Helmholtz instability, adaptive choice of layers.

AMS subject classifications : $35 \mathrm{~L} 45,76 \mathrm{~B} 15,76 \mathrm{~B} 55,65 \mathrm{M} 08$

Institut für Geometrie und Praktische Mathematik RWTH Aachen

Templergraben 55, D-52056 Aachen (Germany)

† Universidad de Málaga

† RWTH Aachen, e-mail: frings@igpm-rwth-aachen.de

* Politechnico di Torino 


\title{
On the hyperbolicity of two- and three-layer shallow water equations
}

\author{
M. Castro ${ }^{\dagger}$, J.T. Frings ${ }^{\ddagger}$, S. Noelle ${ }^{\ddagger}$, C. Parés ${ }^{\dagger}$, G. Puppo ${ }^{\star}$ \\ RWTH Aachen ${ }^{\ddagger}$, Universidad de Málaga ${ }^{\dagger}$, \\ Politecnico di Torino \\ E-mail: frings@igpm.rwth-aachen.de
}

\begin{abstract}
The two-layer shallow water system looses hyperbolicity if the magnitude of the shear velocity is above a certain threshold, essentially determined by the density difference between the two layers. Introducing an additional third layer might recover hyperbolicity in regions of strong shear. We demonstrate that this adaptive two/three-layer approach can cure some of the shortcomings of the two-layer model.
\end{abstract}

\section{Introduction}

In this paper we consider stratified flows. An example which has recently found attention in applied mathematics is the superimposition of the Mediterranean and the Atlantic in the Strait of Gibraltar, e.g. [4]. This layering persists as long as the velocity difference (shear velocity) is not too large. Under this condition the flow may be modeled by two coupled shallow water models, yielding a non-conservative hyperbolic system in one or two space dimensions which may be solved efficiently and accurately by well-balanced finite volume schemes, e.g. [3].

In regions of strong gradients in the bottom topography, however, the lower layer Mediterranean water may accelerate, causing a strong shear and hence a Kelvin-Helmholtz instability. Mathematically, the hyperbolicity of the two-layer model is lost, and usually the finite volume solvers break down or produce questionable results. The obvious, but expensive, solution would be to switch to the full 2D or 3D Navier-Stokes equations and apply the respective free surface incompressible solvers.

A challenging question is whether one can model such local instabilities without giving up the depth-averaging everywhere. Several authors have suggested such models, e.g. LeVeque and Kim [6], Castro and Pares [5], Bouchut and Morales [2] and Audusse [1].

Here we explore an adaptive two/three layer model. We introduce an intermediate third layer to regain hyperbolicity in case it is lost. 


\section{Multilayer flows}

The multilayer shallow water system The flow of $i=1, \ldots, N$ layers of water, ordered from top to bottom, is governed by

$$
\begin{aligned}
& \partial_{t} h_{i}+\partial_{x} q_{i}=0 \\
& \partial_{t} q_{i}+\partial_{x}\left(q_{i}^{2} / h_{i}+g / 2 h_{i}^{2}\right)=-g h_{i} \partial_{x}\left(\sum_{j=1}^{i-1} r_{j i} h_{j}+\sum_{j=i+1}^{N} h_{j}+b\right)
\end{aligned}
$$

with densities $\rho_{i}<\rho_{i+1}$ and $r_{i j}:=\frac{\rho_{i}}{\rho_{j}}$ denoting the density ratios. The coordinate $x$ refers to the spatial position, $t$ is the time, and $g$ is gravity. The unknowns $q_{i}(x, t)$ and $h_{i}(x, t)$ represent respectively the discharge and the height, i.e. thickness of the $i$-th layer at the section of coordinate $x$ at time $t$. The bottom topography is given by the function $b$.

The system (2.1) can be written in quasi-linear form

$$
w_{t}+\mathcal{A}(w) w_{x}=S(w) b_{x},
$$

cf. [3]. The eigenvalues of matrix $\mathcal{A}(w)$ may become complex even for physically relevant data, corresponding to the development of shear instabilities. We will call a multilayer state $w$ hyperbolic, if the matrix $\mathcal{A}(w)$ is diagonalizable and all its eigenvalues are real.

Loss of hyperbolicity In the two-layer case, the matrix $\mathcal{A}$ becomes a $4 \times 4$ matrix. An approximation formula for the eigenvalues in the limit of almost constant water level, almost equal densities and vanishing velocity difference is given in [7]. The authors distinguish two pairs of eigenvalues, the internal and the external ones:

$$
\begin{aligned}
& \lambda_{\text {ext }}^{ \pm}=\frac{u_{1} h_{1}+u_{2} h_{2}}{h_{1}+h_{2}} \pm\left(g\left(h_{1}+h_{2}\right)\right)^{\frac{1}{2}}, \\
& \lambda_{\text {int }}^{ \pm}=\frac{u_{1} h_{2}+u_{2} h_{1}}{h_{1}+h_{2}} \pm\left[g^{\prime} \frac{h_{1} h_{2}}{h_{1}+h_{2}}\left(1-\frac{\left(u_{1}-u_{2}\right)^{2}}{g^{\prime}\left(h_{1}+h_{2}\right)}\right)\right]^{\frac{1}{2}},
\end{aligned}
$$

where $g^{\prime}:=\left(1-r_{12}\right) g$ is a small constant in this limit. The external eigenvalues $\lambda_{\text {ext }}^{ \pm}$correspond to the eigenvalues of a single-layer shallow water system with total water height $H:=h_{1}+h_{2}$ and an averaged total velocity $u=\frac{u_{1} h_{1}+u_{2} h_{2}}{h_{1}+h_{2}}$. These eigenvalues are therefore distinct and real, thus they are not important regarding the hyperbolicity. If

$$
\kappa:=\frac{\left(u_{1}-u_{2}\right)^{2}}{g^{\prime}\left(h_{1}+h_{2}\right)}>1,
$$

the approximative internal eigenvalues become complex. In [5] it is shown that (2.5) is a good approximative criterion for the loss of hyperbolicity when $r_{12}$ is close to 1 . The ratio $\kappa$ can be interpreted as the 
balance between the stabilizing influence of the difference in density and the destabilizing one of the velocity shear.

We quantify this loss of hyperbolicity by the indicator $\delta$ as follows:

Definition 1. The hyperbolicity indicator $\delta$ for a state $U$ is given by

$$
\delta(U):=\left|\Re\left(\lambda_{\text {int }}^{+}(U)\right)-\Re\left(\lambda_{\text {int }}^{-}(U)\right)\right|-\left|\Im\left(\lambda_{\text {int }}^{+}(U)\right)-\Im\left(\lambda_{\text {int }}^{-}(U)\right)\right|,
$$

where $\Re$ denotes the real part and $\Im$ the imaginary part.

This indicator is nonnegative if the eigenvalues are real and negative if they are complex conjugate. It is extended to the multilayer case by applying it to each pair of internal eigenvalues, then taking the minimum.

\section{The three-layer adaptation}

The breakdown of hyperbolicity corresponds to a Kelvin-Helmholtz instability and the onset of mixing [7]. Our interest is to model this situation in the context of shallow water models. We introduce an intermediate layer around the original interface with constant values for velocity and density attached to it, assuming that all the possible turbulence and mixing is contained within this layer and does not affect the horizontal velocities of the other layers in a substantial way.

A strategy for choosing the intermediate layer Given a twolayer state $\left[\left(\rho_{1}, h_{1}, u_{1}\right),\left(\rho_{2}, h_{2}, u_{2}\right)\right]$ we are now looking at a way to find a corresponding three layer state $\left[\left(\tilde{\rho}_{1}, \tilde{h}_{1}, \tilde{u}_{1}\right),\left(\rho_{m}, h_{m}, u_{m}\right),\left(\tilde{\rho}_{2}, \tilde{h}_{2}, \tilde{u}_{2}\right)\right]$ such that the discharge $(\mathrm{Q})$, mass $(\mathrm{M})$ and total water height $(\mathrm{H})$ are conserved:

$$
\begin{aligned}
\sum_{i} \rho_{i} h_{i} u_{i} & =\sum_{i} \tilde{\rho}_{i} \tilde{h}_{i} \tilde{u}_{i}+\rho_{m} h_{m} u_{m} \\
\sum_{i} \rho_{i} h_{i} & =\sum \tilde{\rho}_{i} \tilde{h}_{i}+\rho_{m} h_{m} \\
\sum_{i} h_{i} & =\sum_{i} \tilde{h}_{i}+h_{m}
\end{aligned}
$$

In the upper and lower layer the densities and the velocities are set to

$$
\tilde{u}_{1}=u_{1}, \quad \tilde{u}_{2}=u_{2}, \quad \tilde{\rho_{1}}=\rho_{1}, \quad \tilde{\rho_{2}}=\rho_{2} .
$$

Next, among several possible choices we pick $\rho_{m}=\frac{1}{2}\left(\rho_{1}+\rho_{2}\right)$ in the intermediate layer, thus enforcing constant density throughout the layer, and consider $h_{m}$ a free parameter. This gives

$$
\tilde{h}_{1}=h_{1}-\frac{1}{2} h_{m}, \quad \tilde{h}_{2}=h_{2}-\frac{1}{2} h_{m}, \quad u_{m}=\frac{\rho_{1} u_{1}+\rho_{2} u_{2}}{\rho_{1}+\rho_{2}} .
$$

The density $\rho_{m}$ is independent of $x$ and $t$. Thus even for multiple adaption steps the three-layer context persists, simplifying the construction of an adaptive solver. 
Hyperbolic domains We test the hyperbolicity of 10.000 randomly chosen two-layer test states and compare with those of the corresponding three-layer states gained by the transformation formula $(\mathrm{C})$. The ratio $r_{12}=0.98$ is kept fix.

The hyperbolicity is tested by inserting the test states into the matrix $\mathcal{A}$ in (2.2) and then numerically calculating the eigenvalues. In case of all real, distinct eigenvalues we plot a blue dot in the left graph in Fig. 3.1, otherwise we plot a red dot in the right graph. The axes are $\left(u_{2}-u_{1}\right)^{2}$ and $h_{1}+h_{2}$. With this choice, the hyperbolic and the non-hyperbolic region are approximately ${ }^{1}$ separated by the straight line $\kappa=1$.
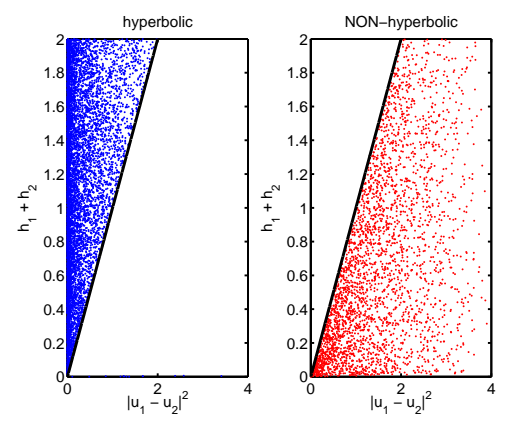

Figure 3.1: Hyperbolic domain for 2-layer test cases, $r_{12}=0.98$
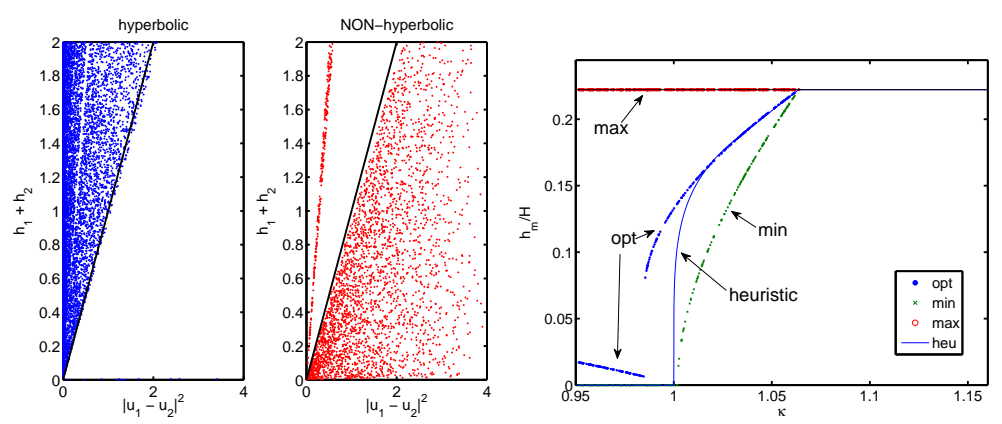

Figure 3.2: Left: Hyperbolic domain for 2-3-layer adaptation, $\Theta=0.111$; Right: $h_{m}^{o p t}, \mathcal{H}^{o p t}$ and heuristic $\tilde{h}_{m}^{\text {heu }}$

To check whether we gain hyperbolicity with our adaptation strategy, we try to find any intermediate layer height for the given test states

\footnotetext{
${ }^{1}$ Note that there are indeed some hyperbolic states with $\kappa>1$ close to the approximative boundary. Also, there is a second region of hyperbolicity for very large shear velocities, but this is not in the focus of our work.
} 

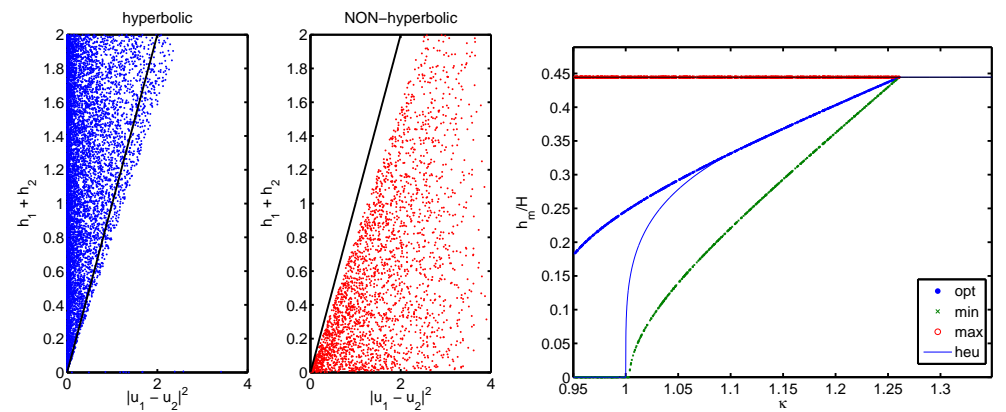

Figure 3.3: Left: Hyperbolic domain for 2-3-layer adaptation, $\Theta=0.222$; Right: $h_{m}^{\text {opt }}, \mathcal{H}^{\text {opt }}$ and heuristic $\tilde{h}_{m}^{\text {heu }}$
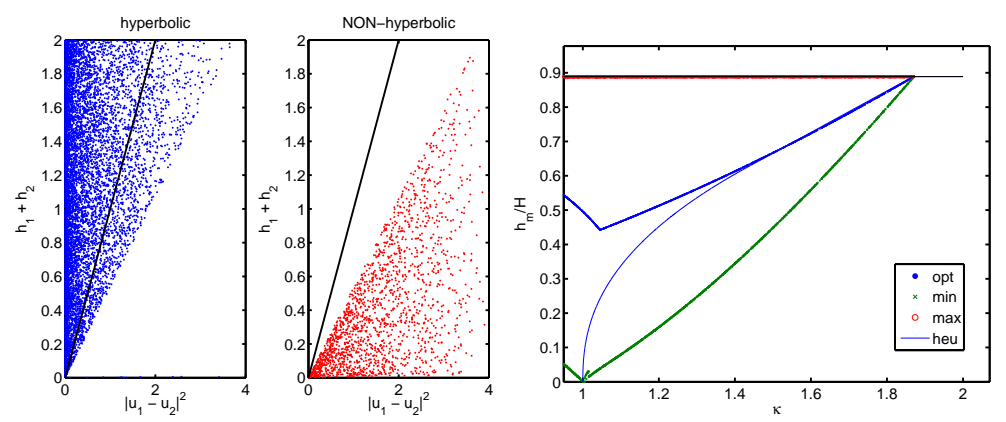

Figure 3.4: Left: Hyperbolic domain for 2-3-layer adaptation, $\Theta=0.444$; Right: $h_{m}^{o p t}, \mathcal{H}^{o p t}$ and heuristic $\tilde{h}_{m}^{\text {heu }}$

that gives a hyperbolic state with three positive layer heights. The intermediate layer height $h_{m}^{o p t}$ is chosen to maximize the indicator $\delta$ among all states with positive layer heights. Again we plot dots indicating the hyperbolicity, this time of the three-layer system, but at the location given by the values of the associated original two-layer system. Thus we can observe from the plots the change in hyperbolicity triggered by the two/three-layer adaptation. We can indeed notice a gain in hyperbolicity but a clear picture only emerges if the data are ordered by the ratio $\Theta:=\frac{h_{2}}{H}$ with $H:=h_{1}+h_{2}$ the total water height. The plots on the left in Figs. 3.2, 3.3 and 3.4 show the gain for different values of $\Theta$. Note that there is a small area in Fig. 3.2 where the hyperbolicity is lost when transferring from two to three layers. This does not pose a problem as those states are hyperbolic for the two-layer model. 
Choice of intermediate layer height We fix $\Theta$ and randomly generate a set with 10000 two-layer test states with $h_{2} / H=\Theta$. For each test state, using the transformation rules $(\mathrm{C})$, we search for a three-layer state with all positive layer heights that maximizes $\delta$. This gives an intermediate height $h_{m}^{o p t}$. Now plotting $\left(\kappa, h_{m}^{o p t} / H\right)$ we found interesting structures as shown by the exemplary results in the plots on the right of Figs. 3.2, 3.3 and 3.4. $\Theta$ is fixed for each plot, but varies from plot to plot. Considering several values of $\Theta$, the points $\left(\kappa, h_{m}^{o p t} / H\right)$ seem to form a surface $h_{m}^{o p t}(\kappa, \Theta)$. Note that for each $\Theta$, there is a value $\kappa_{\max }$ such that for $\kappa>\kappa_{\max }$ the third layer cannot cure the hyperbolicity loss, at least not without deteriorating to a two-layer state again.

There are three additional alleged curves in these plots. The two sets of pairs $\left(\kappa, h_{m}^{\min / \max }\right)$ are defined as follows: define $\mathcal{H}^{\text {opt }}$ for a given $\kappa$ as the largest interval of hyperbolic points containing $h_{m}^{o p t}$. This interval exists as the eigenvalues depend smoothly on the state (and thus on $h_{m}$ ). Then $\mathcal{H}^{\text {opt }}=:\left[h_{m}^{\min }, h_{m}^{\max }\right]$. The remaining curve is given by a heuristic approach for the intermediate layer height.

Heuristic intermediate layer height As a result of the structure revealed by the Figs. 3.2, 3.3 and 3.4 we can define a heuristic method to determine $h_{m}$. We fit curves to the point values of $h^{o p t}$ given for different fixed values of $\Theta$ and then use interpolation between the discrete curves to get a function $\tilde{h}_{m}^{\text {heu }}$ depending on $\Theta$ and $\kappa$. The resulting function $h_{m}^{\text {heu }}$ is defined only for $1<\kappa<\kappa_{\max }$, i.e. for those values of $\kappa$ for which hyperbolicity could be regained. $h_{m}^{\text {heu }}$ is shown in the figures mentioned above as solid lines lying within the optimal intervals $\mathcal{H}^{\text {opt }}$ found for the tested states. In the interval $\left[\left(\kappa_{\max }+1\right) / 2, \kappa_{\max }\right)$ it is defined as a leastsquares linear fit to the optimal heights. In the interval $\left[1,\left(\kappa_{\max }+1\right) / 2\right)$ the definition is $h_{m}^{\text {heu }}(\kappa, \Theta):=c_{\Theta}(1-\kappa)^{\alpha_{\Theta}}$, where $c_{\Theta}, \alpha_{\Theta}>0$ are chosen in such a way that the linear fit in $\kappa_{\max } / 2$ is matched with $\mathcal{C}_{1}$ smoothness. For $\kappa=1$ it connects to the corresponding two-layer hyperbolic state.

Loss of hyperbolicity for a two-layer tidal flow over a hump We present the details of a tidal flow over a hump as shown in Fig. 3.5. The initial data are set to:

$$
\begin{aligned}
\eta & :=h_{1}+h_{2}+b=2, & h_{2} & =1+0.45 \cdot \operatorname{atan}(1.5(x+0.75))-b, \\
q_{1} & =0.001, & q_{2} & =-0.001, \\
b & =e^{-x^{2}} & &
\end{aligned}
$$

with $r=0.98$ and $g=9.812$. The spatial domain is $I=(-3,3)$ subdivided into 20 cells and CFL-Number is $\mathrm{cfl}=0.8$. Absorbing boundary conditions are applied. 

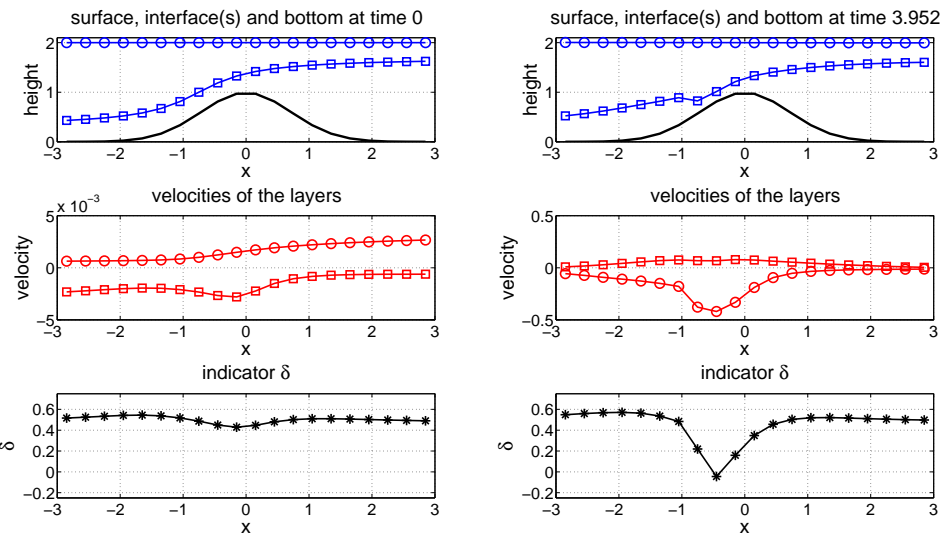

Figure 3.5: Left: Initial state; Right: Situation close to loss of hyperbolicity
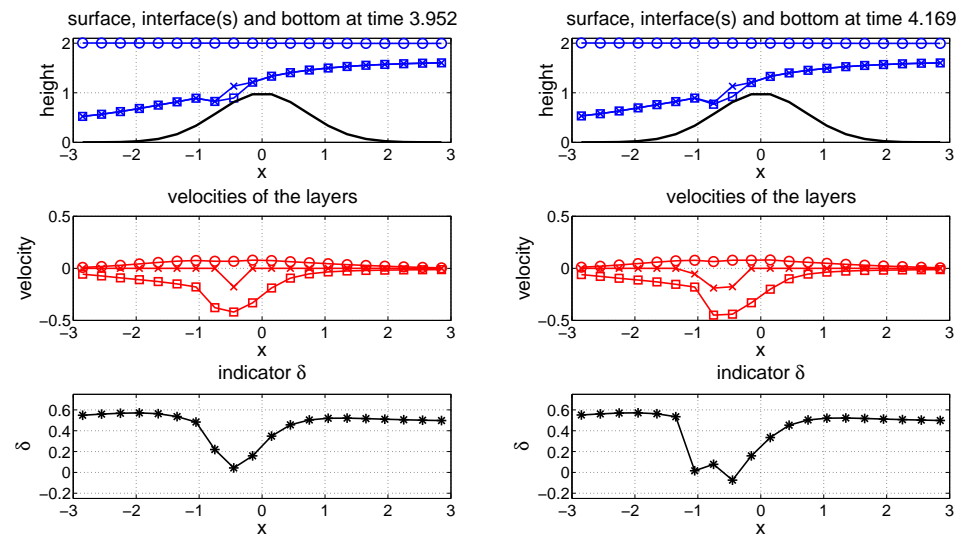

Figure 3.6: Left: Refined state, hyperbolicity regained; Right: Situation close to second loss of hyperbolicity

This test problem is an idealized version of a natural two-layer flow being found e.g. at the Strait of Gibraltar, where the denser Mediterranean Sea forms the lower layer and "spills" over a hump while being moved by tidal forces. Our goal is to design an efficient numerical solver that retains most of the physical features of the real flow.

The simulation runs up until time $t=3.9524$, where the indicator 
shows that hyperbolicity is close to being lost, see Fig. 3.5. At this time an adaptation step is carried out using our heuristic approach to determine the intermediate height. The simulation then continues to run for some time steps until time $t=4.1689$ when the three-layer system loses hyperbolicity in the same cell that was marked critical before the adaptation step, see Fig. 3.6. This is caused mainly by the transport of the intermediate layer. Mass is flowing away from the formerly critical cell and the intermediate layer height drops below $h_{m}^{\text {min }}$ for that state.

The adaptive strategy we have designed can cure hyperbolicity at a given fixed time. However, our results suggest that the cure may not be stable under time evolution. Several improvements are possible, and they will be the subject of future research.

\section{References}

[1] E. Audusse, A multilayer Saint-Venant model: derivation and numerical validation., Discrete Contin. Dyn. Syst. Ser. B, 5(2):189214, 2005.

[2] F. Bouchut and T. Morales, An entropy satisfying scheme for twolayer shallow water equations with uncoupled treatment., M2AN Math. Model. Numer. Anal., 42(4):683-698, 2008.

[3] M. Castro, J. M. Gallardo, and C. Parés, High order finite volume schemes based on reconstruction of states for solving hyperbolic systems with nonconservative products. Applications to shallow-water systems., Math. Comp. 75(255):1103-1134, 2006.

[4] M. Castro, J. Macías, and C. Parés. A Q-scheme for a class of systems of coupled conservation laws with source term. Application to a two-layer 1-D shallow water system., M2AN Math. Model. Numer. Anal, 35(1):107-127, 2001.

[5] M. J. Castro Daz, E. D. Fernndez-Nieto, J. M. Gonzlez-Vida and C. Pars. Numerical treatment of the loss of hyperbolicity of the two-layer shallow-water system., Submitted to Journal of Scientific Computing.

[6] J. Kim and R. J. LeVeque, Two-layer shallow water system and its applications, Proceedings of the Twelth International Conference on Hyperbolic Problems, Maryland 2008.

[7] J. B. Schijf and J. C. Schonfeld. Theoretical considerations on the motion of salt and fresh water., in: Proceedings of the Minn. Int. Hydraulic Conv., Joint Meeting IAHR Hydro. Div. ASCE. (September 1953), pp. 321-333, 1953. 